\title{
Uso de tecnologías de información y comunicación en el proceso de formación de estudiantes de contaduría pública
}

\author{
Use of information and communication technologies in the training process of students of \\ public accounting
}

\begin{tabular}{l|r}
\cline { 2 - 2 } & Génesis Rojas \\
\hline $\begin{array}{l}\text { Artículo recibido en enero 2019 } \\
\text { Arbitrado en febrero 2019 } \\
\text { Publicado en mayo 2019 }\end{array}$ & genesis.rojas.12@gmail.com \\
& ORCID: 0000-0002-3447-826X \\
& Universidad de Carabobo, Venezuela \\
\hline
\end{tabular}

RESUMEN

Palabras clave:
La presente investigación tiene como objetivo proponer el uso de las Tecnologías de Información y Comunicación (TIC) como herramientas de enseñanza en el proceso de formación, para complementar el perfil de los estudiantes egresados de Contaduría Pública de la Facultad de Ciencias Económicas y Sociales de la Universidad de Carabobo, Venezuela. Este estudio reúne las características de una investigación con un nivel comprensivo, bajo la modalidad de proyecto factible, sustentado en un estudio descriptivo con un de diseño de campo no experimental Fue posible concluir que en la Facultad de Ciencias Económicas y Sociales existe una necesidad latente de incluir en el pensum, mayor contenido de las tecnologías de información y comunicación para complementar su currículo, ya que en la actualidad, la mayoría de las empresas utilizan estas herramientas para realizar sus operaciones financieras y los estudiantes de Contaduría Pública necesitan estar mejor preparados en esa área y tener mayor competencia en el campo laboral.

Tecnologías de Información y Comunicación, pensum, perfil del egresado
ABSTRACT

Keywords:
The purpose of this research is to propose the use of Information and Communication Technologies (ICTs) as teaching tools in the training process, to complement the profile of students graduated from Public Accounting of the Faculty of Economics and Social Sciences from the University of Carabobo, Venezuela. This study combines the characteristics of a research with a comprehensive level, under the modality of a feasible project, based on a descriptive study with a non-experimental field design. It was possible to conclude that in the faculty of economic and social sciences there is a latent need to include in the curriculum, greater content of information and communication technologies to complement their education, since at present most of the companies use these tools to perform their financial operations, and public accounting students need to be better prepared in that area, and have more competence in the labor field.

Information and Communication Technologies, pensum, profile of the graduate 


\section{INTRODUCCIÓN}

La sociedad ha evolucionado y de ella ha surgido profesionales más eficientes, que exigen muy buena información, dado esto es racional pensar que el proceso contable y el profesional encargado de este, también cambien para atender esta nueva necesidad; el contador público debe tener habilidades y conocimientos, acordes a la nueva era o "sociedad digital".

Por su parte, los sistemas contables están compuestos por cuatro elementos, un emisor (Contador público); un medio (canal por donde se dirige el mensaje); el mensaje (es el conjunto de datos que producen información) y los usuarios (son aquello receptores, denominados partes de interés). En este caso, la tecnología afecta el medio por el cual circulan los datos para producir información y posterior mente conocimiento, pero la contabilidad va más allá, es: un sistema de información, que captura, procesa, almacena y distribuye un tipo particular de datos, la información financiera, jurídica y socio-económica, vital para la correcta toma de decisiones en la empresa. O más avanzado; un sistema de información y comunicación para la toma de decisiones de las partes de interés, por consiguiente, interactúan canales de comunicación.

El proceso contable según Flores (2004)

Está compuesto por cinco macro procesos; reconocimiento de los hechos; sistematización o procesamiento; revelación; análisis y control; dentro del primero se encuentran las actividades de identificar, clasificar, valuar; en el segundo, registrar, asignar, verificar y ajustar; en el tercero revelar, en el cuarto y quinto son retroalimentaciones al proceso. (P.87)

Después de ilustrar el proceso, se observa el impacto de las tecnologías en el que hacer contable. Las tecnologías de la información operan como motor del cambio que permite dar respuesta a las nuevas necesidades de información. Cabe aclarar, que las tecnologías minimizan el trabajo del profesional para incrementar la calidad de vida de este, en el caso del contador público se automatiza el proceso contable, permitiendo que éste se dedique a los procesos de análisis y control. En general la automatización de los procesos produce mejores condiciones para la organización porque agiliza los procesos, ahorra tiempo y reduce costos.

De tal modo, que las tecnologías de información y comunicación (TIC) según Gil (2002, 25), constituyen "un conjunto de aplicaciones, sistemas, herramientas, técnicas y metodologías asociadas a la digitalización de señales analógicas, sonidos, textos e imágenes, manejables en tiempo real". Como señala este autor, las TIC son herramientas, por lo tanto, se debe hacer uso de ellas para complementar los conocimientos adquiridos en la carrera de Contaduría Pública, ya que está a disposición de todo el que desee utilizarlas. Por su parte, Ochoa y Cordero $(2002,76)$, establecen que 
"son un conjunto de procesos y productos derivados de las nuevas herramientas (hardware y software), soportes y canales de comunicación, relacionados con el almacenamiento, procesamiento y la transmisión digitalizada de la información".

Asimismo, Thompson $(2004,48)$ define las tecnologías de información y comunicación, como "aquellos dispositivos, herramientas, equipos y componentes electrónicos, capaces de manipular información que soportan el desarrollo y crecimiento económico de cualquier organización". Cabe destacar que en ambientes tan complejos como los que deben enfrentar hoy en día las organizaciones, sólo aquellos que utilicen todos los medios a su alcance, y aprendan a aprovechar las oportunidades del mercado visualizando siempre las amenazas, podrán lograr el objetivo de ser exitosas.

Por su parte, Marqués (2000) destaca que las TIC, apuntan a un nuevo paradigma para la enseñanza que se va perfilando en el marco de la sociedad de la información con las nuevas prestaciones de los ordenadores, la telefonía, los "más media" $y_{\text {, }}$ especialmente, Internet, que proporcionan acceso a todo tipo de información, siempre disponible en todas partes, y facilitan canales de comunicación también inmediatos.

A tal efecto, Casanova $(2002,58)$ define las TIC como: "todos aquellos artefactos (tanto fijos como móvil) que permiten al gestión y la transmisión de la información a través de la informática, sin la necesidad por parte del usuario final de trasladarse a un punto geográfico específico". Este autor señala que los alumnos desde su computadora podrán investigar y estudiar, en la escuela, en los centros de informática, o en su propio hogar, sin necesidad de hacer un viaje hasta la biblioteca a consultar estos textos o adquirirlos en tiendas especializadas. Las TIC se están posicionando día a día en el ámbito de la vida de la sociedad contemporánea, siendo de gran ayuda para la obtención de la información y el desarrollo de la comunicación entre las personas.

De esta manera, el proceso de globalización, el conocimiento y la innovación tecnológica juegan un papel capital en las actividades económicas y, por supuesto, en el desarrollo de las naciones. El Contador Público Independiente no puede obviar el acompañamiento tecnológico que facilita su ingreso al mercado laboral, de allí la importancia de las TIC como herramienta de enseñanza: Internet, correo electrónico, entre otras para el manejo de programas $y$ sistemas que influirán en la calidad y eficiencia de la educación y formación de los estudiantes de contaduría.

En este sentido, la contabilidad como sistema de información se ha adaptado a las necesidades de sus usuarios, pero su desarrollo ha estado limitado por los recursos tecnológicos. Los Contadores se preguntan qué información demandan los usuarios. Los directivos de las empresas demandan estados financieros mensuales, los cuales en el pasado se realizaban de forma manual; sin embargo, hoy en día cualquier software especializado permite 
hacer cierres automatizados de la contabilidad pulsando una tecla.

Es por ello, la importancia para el Contador conocer los avances tecnológicos en la actualidad. La aplicación de la informática en la contabilidad, facilita los diferentes procesos de la misma como son: el registro de los hechos económicos ocurridos, el procesamiento de datos en los registros, la facturación, las órdenes de pedido, el control de los inventarios, todas las operaciones de cobros y pagos con proveedores y clientes, hasta la elaboración de la información que culmina con la presentación de los estados financieros, instrumentos cuantitativos de gran utilidad para la toma de decisiones en cualquier entidad.

En la esfera contable existen variados softwares adaptables a las necesidades del Contador que posibilitan y facilitan el desarrollo más eficiente de un gran número de operaciones financieras. Aunque los fundamentos de la contabilidad siguen vigentes, y seguirá vigente, lo que no debe seguir vigente es enseñar de forma manual, registrando un asiento contable por cada transacción, porque con los avances tecnológicos esto ha cambiado totalmente y ahora podemos generar a partir de un único ingreso de datos la información de manera integrada en tiempo real que sea confiable y oportuna.

El perfil profesional determina las competencias que requiere el futuro profesional para solventar de manera adecuada y oportuna los problemas y necesidades del entorno, Para Díaz (2005:175), "el perfil profesional lo componen tanto conocimientos y habilidades como actitudes". Entre los programas que un estudiante de Contaduría Pública debe conocer, está el paquete de Microsoft Office en el cual está la hoja de cálculo de Excel, también debe conocer los sistemas integrados, que día a día están siendo utilizados por las mayorías de las empresas que realizan alguna actividad comercial, tanto dentro como fuera del país, y un Contador Público debe conocer estos programas y sus funciones para atender de forma más eficiente las necesidades de sus clientes, ya que las empresas buscan Contadores que no solo estén preparados en el área contable, sino también en el área Tecnológica.

En este sentido, la National School Board Association (2002), plantea existe una creciente conciencia entre los responsables de trazar las políticas educativas, los dirigentes de las empresas y los educadores en general de que el sistema educativo diseñado para preparar a los alumnos para una economía agraria o industrial no brindará a los individuos las habilidades y los conocimientos necesarios para triunfar en la economía y la sociedad del conocimiento del siglo XXI. Los sistemas educativos enfrentan el desafío de transformar el plan de estudios y el proceso de aprendizaje para brindar a los estudiantes las habilidades que les permitan funcionar de manera efectiva en este entorno en constante cambio. 
La economía mundial basada en la tecnología también presenta otros desafíos para los países a medida que las economías nacionales se tornan más dependientes del ámbito internacional, lo que trae aparejado un creciente intercambio de información, tecnología, productos, capital e individuos entre las naciones. Este nuevo entorno económico dará lugar a una nueva era de competencia mundial por bienes, servicios y conocimiento. Como consecuencia, muchos países están atravesando transformaciones radicales en sus estructuras políticas, económicas y sociales. En las naciones industrializadas, la economía, anteriormente basada en un modelo industrial, está cambiando hacia una economía basada en la información.

Esta transformación, exige que los docentes adquieran nuevos conocimientos y habilidades; presentando nuevos desafíos para los sistemas educativos en cuanto a cómo brindar a los individuos los conocimientos y habilidades necesarios para triunfar en este nuevo y dinámico entorno de continuos cambios tecnológicos, donde la producción de conocimiento crece a una velocidad cada vez mayor.

Es por ello, que para alcanzar esta meta debe producirse un cambio en la concepción tradicional del proceso de aprendizaje y una nueva comprensión acerca de cómo las nuevas tecnologías digitales pueden ayudar a crear nuevos entornos de aprendizaje en los que los alumnos se sientan más motivados y comprometidos, asuman mayores responsabilidades sobre su propio aprendizaje y puedan construir con mayor independencia sus propios conocimientos.

\section{MATERIALES Y MÉTODO}

Este estudio reúne las características de una investigación con un nivel comprensivo, bajo la modalidad de un Proyecto Factible, puesto que su objetivo es ofrecer solución a un problema real; específicamente en diseñar lineamientos que permitan la actualización del pensum actual de Contaduría Pública, para mejorar el perfil del egresado, mediante el uso de las Tecnologías de Información y Comunicación.

Con base en los objetivos de esta investigación, se diseñó una investigación de campo, no experimental, debido a que los datos de interés son tomados en forma directa de la realidad; sin dejar de hacer énfasis en los datos secundarios, de los que se obtendrá información a través de fuentes confiables. De una población de 805 estudiantes de la carrera de Contaduría Pública de la Universidad de Carabobo, se seleccionó una muestra de 41 individuos, a quienes se les suministró una encuesta con preguntas dicotómicas, cuyos datos obtenidos fueron organizados, tabulados y analizados con técnicas estadísticas.

\section{RESULTADOS Y DISCUSIÓN}

A continuación se presentan las preguntas realizadas a los Estudiantes inscritos en el noveno y décimo semestre de Contaduría Pública, y los resultados obtenidos: 
Tabla № 1 ¿Conoce usted cuales son las tecnologías de la información y comunicación que dan soporte al trabajo del contador público?

\begin{tabular}{ccc}
\hline ALTERNATIVA & FRECUENCIA & PORCENTAJE \\
\hline SI & 16 & 39 \\
NO & 25 & 61 \\
TOTAL & $\mathbf{4 1}$ & $\mathbf{1 0 0} \%$ \\
\hline
\end{tabular}

Fuente: Rojas, G (2018)

Este resultado deja en evidencia la deficiencia de conocimiento de las TIC que existe entre los estudiantes de los últimos semestres de contaduría Pública; ya que, solo un 39 por ciento conoce las tecnologías de información y comunicación que dan soporte al trabajo del contador público.

Tabla $\mathbf{N}^{\circ} 2$ ¿Cree usted que las tecnologías de la información y comunicación son necesarias para el Contador Público en la actualidad?

\begin{tabular}{ccc}
\hline ALTERNATIVA & FRECUENCIA & PORCENTAJE \\
\hline SI & 40 & 98 \\
NO & 1 & 2 \\
TOTAL & $\mathbf{4 1}$ & $\mathbf{1 0 0 \%}$ \\
\hline
\end{tabular}

Fuente: Rojas, G (2018)

Según los datos obtenidos se determinó que casi en su totalidad, los estudiantes encuestados están de acuerdo que las tecnologías de la información y comunicación son necesarias para el contador público, por lo que son herramientas útiles para mejorar el desempeño del Contador en el área laboral.

Tabla $\mathbf{N}^{\circ} 3$ ¿Considera usted que la Facultad de Ciencias Económicas y Sociales de la Universidad de Carabobo imparte los conocimientos necesarios en cuanto a las tecnologías de la información y comunicación?

\begin{tabular}{ccc}
\hline ALTERNATIVA & FRECUENCIA & PORCENTAJE \\
\hline SI & 2 & 5 \\
NO & 39 & 95 \\
TOTAL & $\mathbf{4 1}$ & $\mathbf{1 0 0} \%$ \\
\hline
\end{tabular}

Fuente: Rojas, G (2018) 
Se evidencia que el 95 por ciento de los estudiantes considera que la Facultad De Ciencias Económicas y Sociales de la Universidad De Carabobo, no imparte los conocimientos necesarios en cuanto a las tecnologías de la información y comunicación.

Tabla No 4 ¿Sabía usted que la Facultad de Ciencias Económicas Y Sociales de la Universidad de Carabobo, cuenta con laboratorios de informática dotados para dar clases computarizadas de estadísticas, contabilidad, entre otras materias?

\begin{tabular}{ccc}
\hline ALTERNATIVA & FRECUENCIA & PORCENTAJE \\
\hline SI & 12 & 29 \\
NO & 29 & 71 \\
TOTAL & $\mathbf{4 1}$ & $\mathbf{1 0 0} \%$ \\
\hline
\end{tabular}

Fuente: Rojas, G (2018)

Se pudo conocer que el 71 por ciento de los estudiantes si conocían la existencia de los laboratorios de informática que posee la Facultad de Ciencias Económicas y Sociales de la Universidad de Carabobo.

Tabla $\mathbf{N}^{\circ} \mathbf{5}$ ¿Cree Usted que el pensum actual de la carrera de Contaduría Pública de la Facultad de Ciencias Económicas y Sociales de la Universidad De Carabobo necesita ser actualizado?

\begin{tabular}{ccc}
\hline ALTERNATIVA & FRECUENCIA & PORCENTAJE \\
\hline SI & 41 & 100 \\
NO & 0 & 0 \\
TOTAL & $\mathbf{4 1}$ & $\mathbf{1 0 0 \%}$ \\
\hline
\end{tabular}

Fuente: Rojas, G (2018)

Los datos obtenidos demuestran que todos están de acuerdo que el pensum de la carrera de Contaduría Pública de la Universidad de Carabobo necesita ser actualizado. 
Tabla $\mathbf{N}^{\circ} 6$ ¿Los conocimientos recibidos de las tecnologías de la información y comunicación en la Facultad de Ciencias Económicas y Sociales de la Universidad de Carabobo son suficientes para estar preparados en esta área?

\begin{tabular}{ccc}
\hline ALTERNATIVA & FRECUENCIA & PORCENTAJE \\
\hline SI & 1 & 2 \\
NO & 40 & 98 \\
TOTAL & $\mathbf{4 1}$ & $\mathbf{1 0 0 \%}$ \\
\hline
\end{tabular}

Fuente: Rojas, G (2018)

La mayoría de los estudiantes de los últimos semestres de Contaduría Pública, opinan que los conocimientos sobre el uso de las tecnologías de información y comunicación que recibieron durante su formación universitaria, no son suficientes para estar preparados en esa área. Teniendo en cuenta los resultados obtenidos es importante señalar lo que opinan Barreto y Cárdenas $(2011,25)$ respecto a la necesidad de implementar las $\Pi \mathrm{CC}$ en el proceso de enseñanza de la carrera, comentan lo siguiente "en la carrera de Contaduría Pública existe la necesidad de involucrar las TC en su proceso de enseñanza aprendizaje como una actividad permanente para apoyar los procesos curriculares y su renovación e innovación permanente, de cara a las necesidades actuales y futuras de la sociedad y el entorno".

Tabla $\mathbf{N}^{\circ} 7$ ¿Conoce Usted cuales son los programas que le dan soporte al trabajo del Contador Público?

\begin{tabular}{ccc}
\hline ALTERNATIVA & FRECUENCIA & PORCENTAJE \\
\hline SI & 12 & 29 \\
NO & 29 & 71 \\
TOTAL & $\mathbf{4 1}$ & $\mathbf{1 0 0 \%}$ \\
\hline
\end{tabular}

Fuente: Rojas, G (2018)

Para un estudiante de Contaduría Pública es importante conocer cuáles son los Programas que le dan soporte al trabajo del contador público, siendo que un 71 por ciento, no conoce cuales son los programas que pueden ayudarle en el ejercicio de su carrera. 
Tabla No 8 ¿Considera usted que es necesario que un estudiante de Contaduría Pública conozca los programas existentes que facilitan el trabajo del Contador Público?

\begin{tabular}{ccc}
\hline ALTERNATIVA & FRECUENCIA & PORCENTAJE \\
\hline SI & 40 & 98 \\
NO & 1 & 2 \\
TOTAL & $\mathbf{4 1}$ & $\mathbf{1 0 0} \%$ \\
\hline
\end{tabular}

Fuente: Rojas, G (2018)

En este grafico se observa que de la muestra seleccionada, un 98 por ciento de los estudiantes considera necesario que durante el proceso de formación de un contador público, este debe conocer las tecnologías de información y comunicación, que existen hoy en día para facilitar el trabajo del profesional en la Contaduría Pública.

Tabla No 9 ¿Es necesario la inclusión de las tecnologías de la información y comunicación en el contenido del pensum de la carrera de contaduría pública de la Facultad de Ciencias Económicas y Sociales de la Universidad de Carabobo?

\begin{tabular}{ccc}
\hline ALTERNATIVA & FRECUENCIA & PORCENTAJE \\
\hline SI & 41 & 100 \\
NO & 0 & 0 \\
TOTAL & $\mathbf{4 1}$ & $\mathbf{1 0 0 \%}$ \\
\hline
\end{tabular}

Fuente: Rojas, G (2018)

El 100 por ciento de los encuestados están de acuerdo con la afirmación, por lo que se determina es necesario la inclusión de las tecnologías de la información y comunicación en el contenido del pensum de la carrera de contaduría pública de la Facultad de Ciencias Económicas y Sociales de la Universidad de Carabobo, esto confirma que los estudiantes están conscientes que todo está cambiando, y que las organizaciones cada día se apoyan más en las TIC para realizar sus operaciones.

Tabla No 10 Al finalizar la carrera de Contaduría Pública, ¿cree usted que será necesario tener conocimientos adecuados de las tecnologías de la información y comunicación?

\begin{tabular}{ccc}
\hline ALTERNATIVA & FRECUENCIA & PORCENTAJE \\
\hline SI & 41 & 100 \\
NO & 0 & 0 \\
TOTAL & $\mathbf{4 1}$ & $\mathbf{1 0 0 \%}$ \\
\hline
\end{tabular}

Fuente: Rojas, G (2018) 
Los resultados de esta tabla muestran que el noventa y ocho por ciento (98\%) de los estudiantes integrantes de la muestra, ratifican que es necesario tener conocimientos adecuados de las tecnologías de Información Y comunicación.

Tabla No 11 ¿Cree Usted que la Universidad de Carabobo tiene los recursos necesarios para incluir nuevas tecnologías de la información y comunicación?

\begin{tabular}{ccc}
\hline ALTERNATIVA & FRECUENCIA & PORCENTAJE \\
\hline SI & 12 & 30 \\
NO & 29 & 70 \\
TOTAL & $\mathbf{4 1}$ & $\mathbf{1 0 0} \%$ \\
\hline
\end{tabular}

Fuente: Rojas, G (2018)

Según los datos aportados se determinó que el 70 por ciento de los encuestados opinan que la Universidad de Carabobo no cuenta con los recursos necesarios para adaptar el pensum de la carrera con mayor contenido en cuanto al uso de las tecnologías de información y comunicación.

\section{CONCLUSIONES}

El empleo de las TIC como elemento estratégico fundamental en el desarrollo de la enseñanza-aprendizaje, es un elemento importante a tener en cuenta, toda vez que aporta elementos sustanciales que permitirían aprovechar ventajas competitivas acorde con las exigencias del mundo actual

Dentro del contexto educativo, las TIC presentan unas características que resultan muy interesantes para el proceso de enseñanza-aprendizaje, ya que los alumnos son responsables de su aprendizaje, se implican en él, se facilita la interactividad, se motiva al alumnado, se despierta su interés, hay un Feedback continuo entre profesores y alumnos y las herramientas para el aprendizaje son variadas, intuitivas y rápidas. Estas características de las TC provocan que el contexto educativo se caracterice, al mismo tiempo, por los siguientes rasgos: el conocimiento deja de ser lento y escaso, la universidad tiene una nueva función educativa, al igual que los profesores, se replantean las competencias y habilidades y las tecnologías tradicionales dejan de ser las únicas que se implantan en el proceso de enseñanza-aprendizaje.

\section{REFERENCIAS}

Barreto, Oscar, Cárdenas, Sandra (2011). Las Tecnologías de Información y Comunicación en la Formación de Contadores Públicos.Este artículo corresponde al proyecto Las tecnologías de información y comunicación aplicables a los procesos de aprendizaje vol. 12 enero-junio 2011

Casanova, M. (2002). La atención a la diversidad, reto de la innovación 
curricular. Enciclopedia Pedagógica. Tomo 3, pp. 641-655. Universidad Camilo José Cala. España: Editorial Espasa

Dorrego, E. (2004) Transformación de la educación superior en América Latina.En: Nuevas tecnologías y educación. Madrid: EDUTEC/PEARSON

Díaz, Frida (2009) Metodología de Diseño Curricular Para la Educación Superior. 11a edición editorial trillas

Flores, Orlando (2004). Uso de la Tecnología de la Información y la Comunicación (TIC's) en el Desarrollo de las Actividades de los Contadores Públicos del Estado Carabobo.Tesis de Ascenso Para optar a la categoría de profesor asistente. Universidad de Carabobo. Bárbula

Gil, Eva. (2002). Identidad y Nuevas Tecnologías. Disponible en: http:www.voc.edu/web/esplart/gil0902/ht mConsulta: 2016, noviembre 16

Laudon, Kenneth; Laudon, Jane (2012). Sistemas de Información Gerencial. Decimosegunda edición PEARSON EDUCACIÓN, México

Marqués, P. (2000). Las TC y sus aportes a la sociedad. Barcelona: Facultad de Educación, UAB. Departamento de Pedagogía Aplicada. Disponible:
http://dewey.uab.es/PMARQUES/siyedu.h tm

National School Board Association. (2002). Why Change?. Disponible: http://www.nsba.org/sbot/toolkit/WhyCh ange.html

Ochoa, X. y Cordero, S. (2002). Las Nuevas Tecnologías de la Información y la Comunicación. Disponible en: http://www.ruv.itesm.mx/especiales/citela /documentos/material/módulos/módulo s2/contenidoii.htm

Salomon, Gavriel. (1993). Distributed cognitions. Psychological and educational considerations. Cambridge University Press

Spiro, R.J., Coulson, R.L., Feltovich, P.J., y Anderson, D. (1988). Cognitive flexibility theory: Advances knowledge acquisition in ill-structured domains, en V. Patel (ed.), Proceedings of the 10th Annual Conference of the Cognitive Science Society. Hills-dale, NJ: Erlbaum

Thompson, Arthur. (2004). Administración Estratégica. Editorial Mc Graw Hill, México UNESCO (2004). Las tecnologías de la información y la comunicación en la formación docente. Uruguay: Ediciones Trilce 